\title{
TORRES DE VIDRIO DEL CENTRO DE ARTE "REINA SOFÍA" DE MADRID-ESPAÑA
}

\author{
(GLASS TOWERS IN THE ART CENTER “REINA SOFÍA” IN MADRID - SPAIN)
}

\author{
José Luis İñiguez de Onzoño y \\ Antonio Vázquez de Castro, Arquitectos
}

Fecha de recepción: 1-X-91

$691-5$

\section{RESUMEN}

Las torres de ascensores del Centro de Arte "Reina Sofía", destacan en su entorno urbano debido a su importante cerramiento transparente, frente a la opaca fachada del antiguo Hospital Provincial de Atocha.

Las caracteristicas materiales y de funcionamiento de su cerramiento es lo que aquí se expone:

- El sistema de anclaje y fijación ante las acciones gravitatorias y térmicas a través de tirantes verticales de acero, rótulas especiales, y muelles en sus bases.

- El sistema de anclaje y fijación ante acciones horizontales en las esquinas de cada pieza.

- La función de la silicona estructural, como único material de enlace entre paneles para colaborar en la redistribución de esfuerzos.

\section{SUMMARY}

The elevator towers in the Art Center "Reina Sofía" stand out in their urban setting due to their transparent partition against the opaque façade of the old "Hospital Provincial de Atocha".

The material and functional characteristics of their partition are the topic of this article:

- The bracing and fixation system against gravitation and thermic forces through vertical steel ties, special joints and springs in the bases.

- The bracing and fixation system against horizontal actions on the corners of each piece.

- The function of structural silicone as the only material between panels helping in the redistribution of stress.

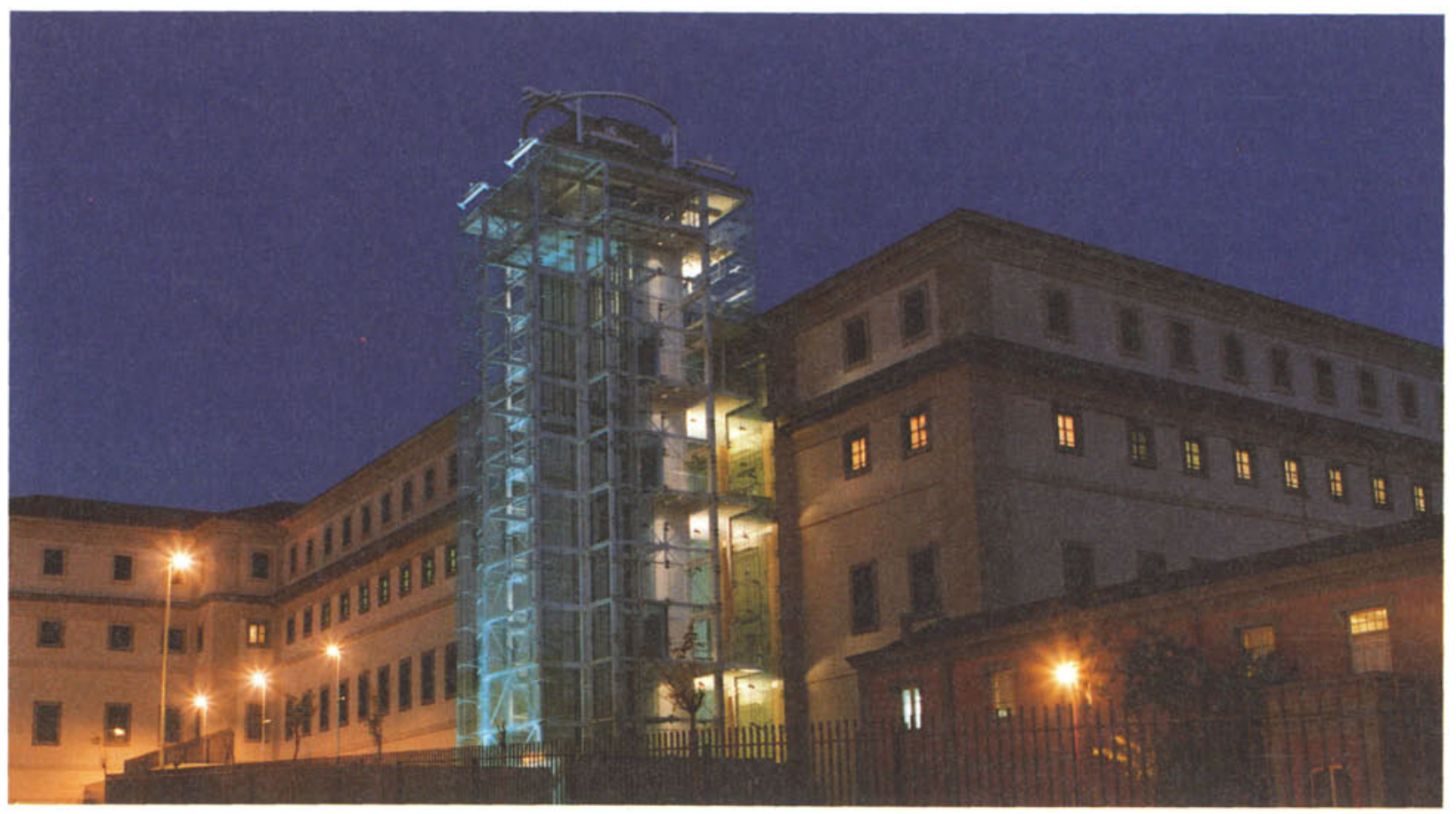

(C) Consejo Superior de Investigaciones Científicas 


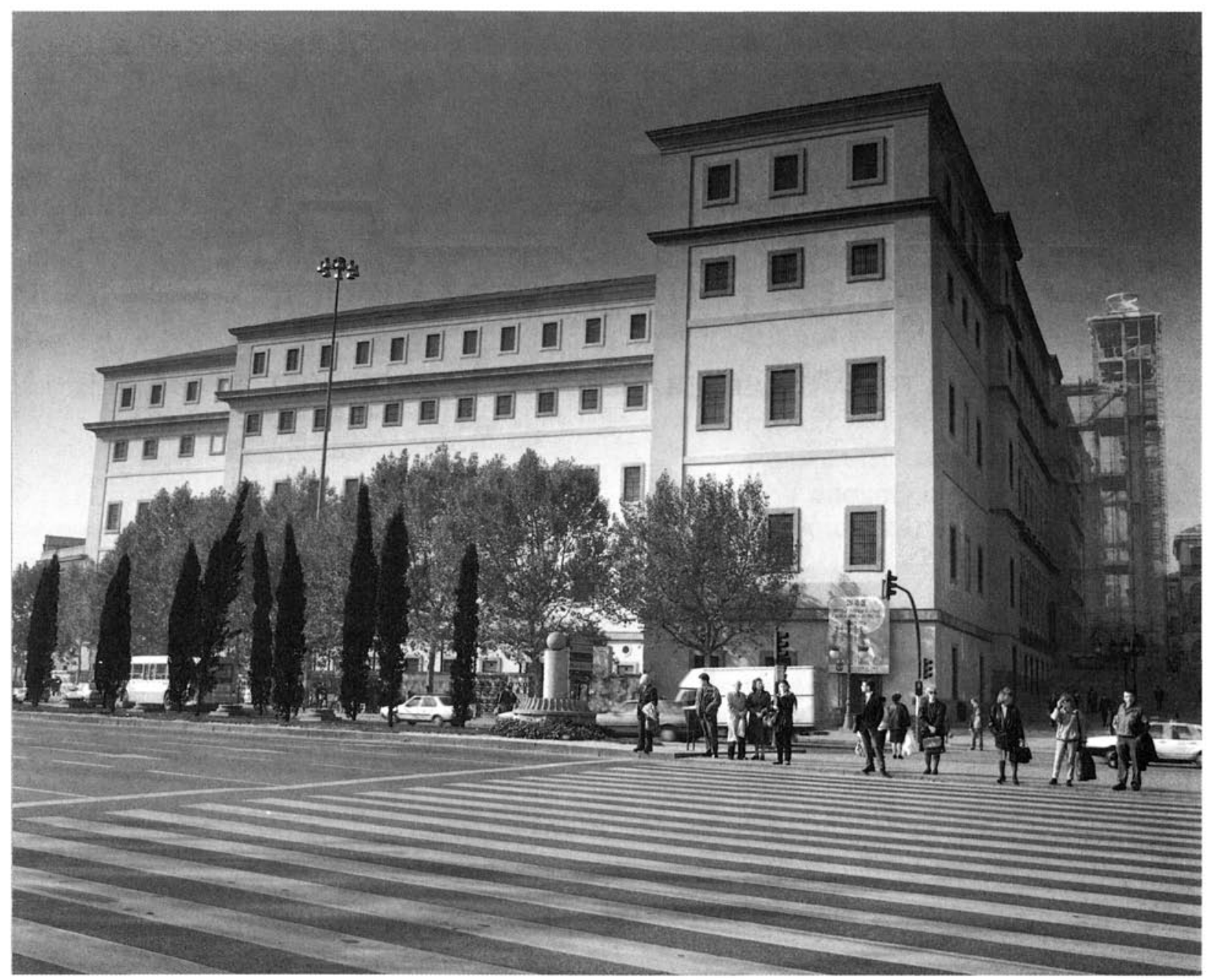

\section{INTRODUCCIÓN}

Dentro del conjunto de obras de remodelación que recientemente se han realizado en el sólido caserón del antiguo Hospital Provincial de Atocha, obra del arquitecto de Carlos III Francisco Sabatini, para completar su transformación en museo de arte moderno, como Centro de Arte "Reina Sofía", se han construido unas torres transparentes de comunicación vertical que han sido adosadas a sus fachadas.

La dinámica del movimiento y la moderna tecnología adoptada en el diseño de las torres enriquecen la serenidad de la antigua construcción en una nueva expresión estilística.

En la fachada principal del edificio, calle de Santa Isabel, y respetando la simetría establecida por el acceso principal se han dispuesto dos torres gemelas que alojan los ascensores panorámicos de público, los cuales conducen el flujo de visitantes de unos niveles del museo a otros.

(c) Consejo Superior de Investigaciones Científicas Licencia Creative Commons 3.0 España (by-nc)
También en una de las fachadas laterales, la de la calle del Hospital, se ha construido una torre de servicio dotada de un amplio montacargas para el trans. porte vertical de las obras de arte, un ascensor de personas y una escalera de servicio.

La singularidad de estas tres torres reside en la total transparencia de sus fachadas, completamente revestidas con paneles de vidrio estructural, y en la cualidad panorámica de sus ascensores, lo que reduce el impacto visual desde el exterior y ofrece magníficas vistas del entorno urbano desde el interior, tanto durante las esperas como durante el desplazamiento en los ascensores.

El objetivo de transparencia y permeabilidad óptica de las fachadas de las torres se ha favorecido con una disposición y diseño estructural caracterizado por la esbeltez de sus elementos y por la propia ubicación de los mismos, alejados de las esquinas. 


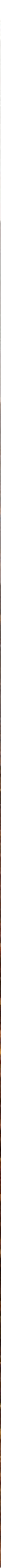




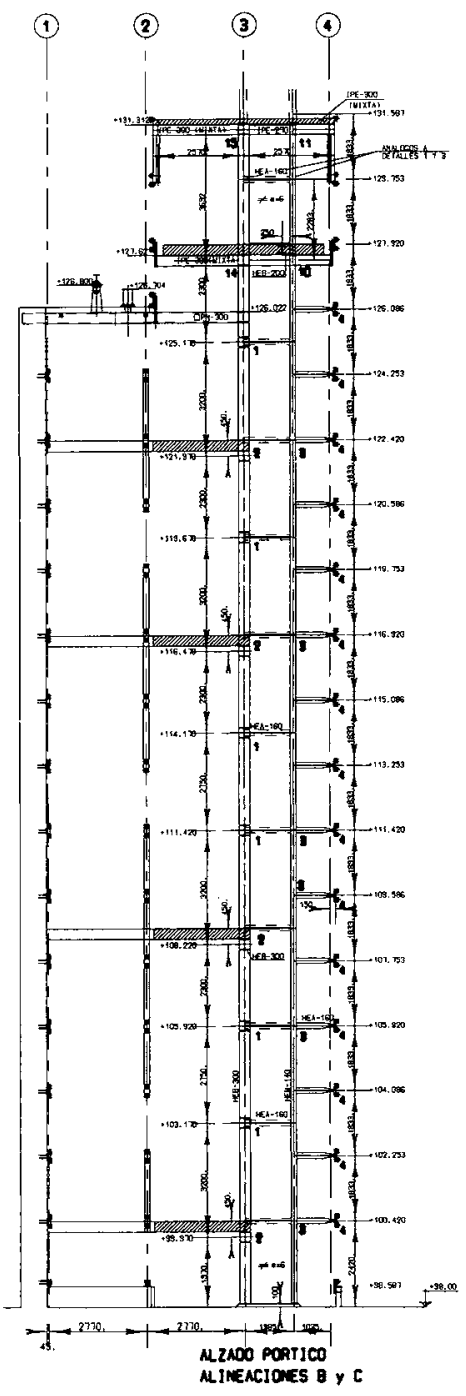

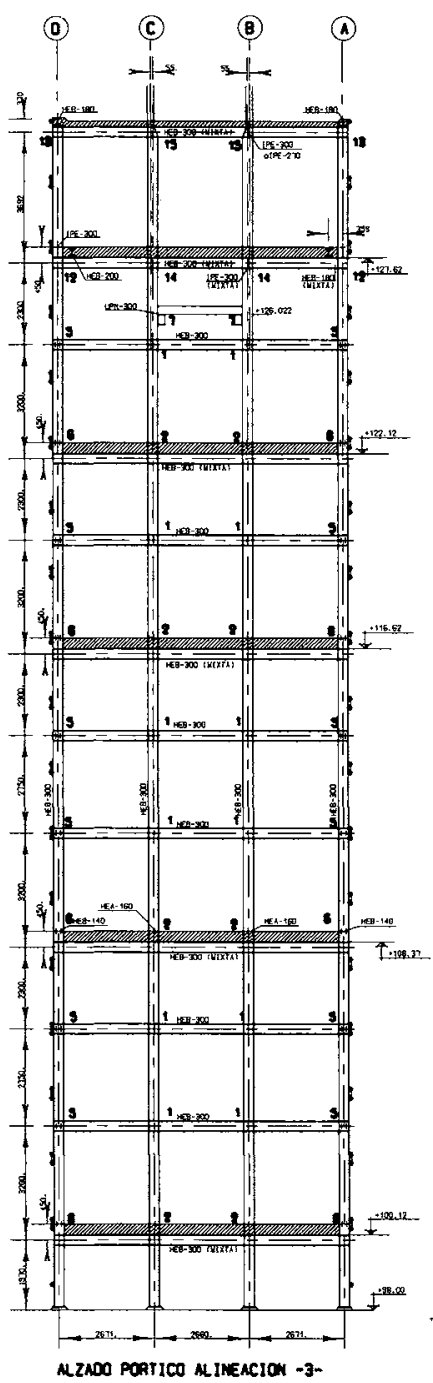

\section{ESTRUCTURA}

La estructura principal de las torres se ha resuelto en acero, en aras de una mayor esbeltez del conjunto, si bien para los elementos horizontales se ha recurrido a losas macizas de hormigón armado que garantizaban un mayor confort ante las cargas dinámicas de la circulación del público.

La espina dorsal de las torres gemelas de visitantes está formada por un pórtico rígido de acero con doce niveles y treinta y siete metros de altura. Los cuatro pilares principales tienen rígidamente conectados otros cuatro soportes satélites, totalmente exentos, cuya misión funcional es la fijación de las guias de los ascensores y de los brazos de conexión con la fachada de vidrio. En seis de los dinteles del pórtico apoyan las plataformas de desembarco de los ascensores, la planta de maquinaria y la cubierta.

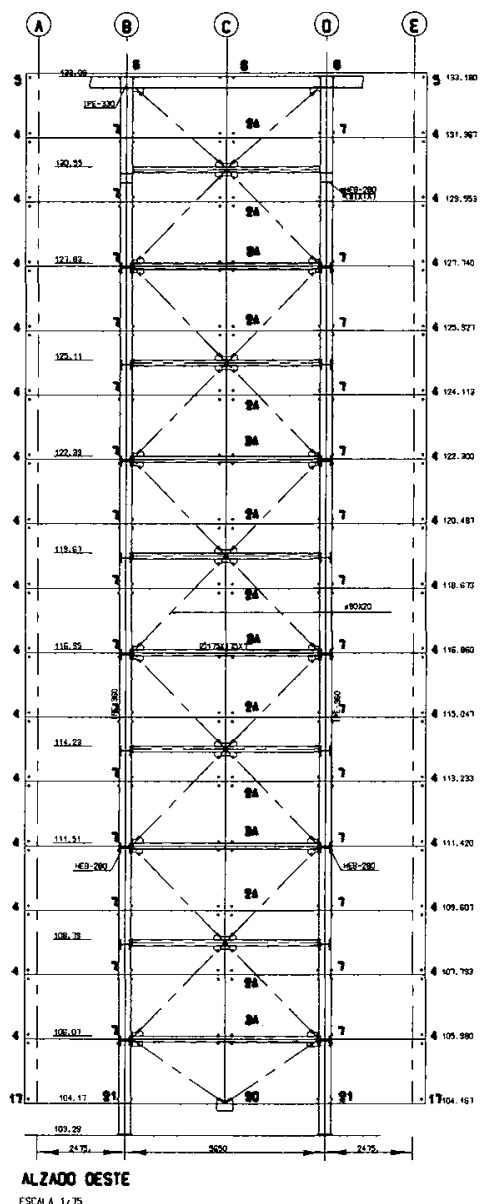

AL2AOO OESTE
La torre de servicios tiene una organización estructural diferente, como consecuencia de sus mayores dimensiones en planta y de su propia ordenación funcional. Además de un pórtico rígido central, dispone de varios planos verticales de arriostramiento formados por barras de acero en cruz de San Andrés.

Sobre el nivel de cubierta de las torres (incluida la de servicios) se organiza una sigular estructura de perfiles tubulares que soporta en voladizo los pescantes de cuelgue de las fachadas, y que sirve también de apoyo a grúas polares que desplazan las góndolas de limpieza de los vidrios.

Los criterios de limitación de deformabilidad de la estructura, bajo las acciones horizontales y de uniformidad geométrica de los elementos repetitivos en toda la altura de las torres, han determinado el dimensionado de los perfiles de la estructura metálica. 


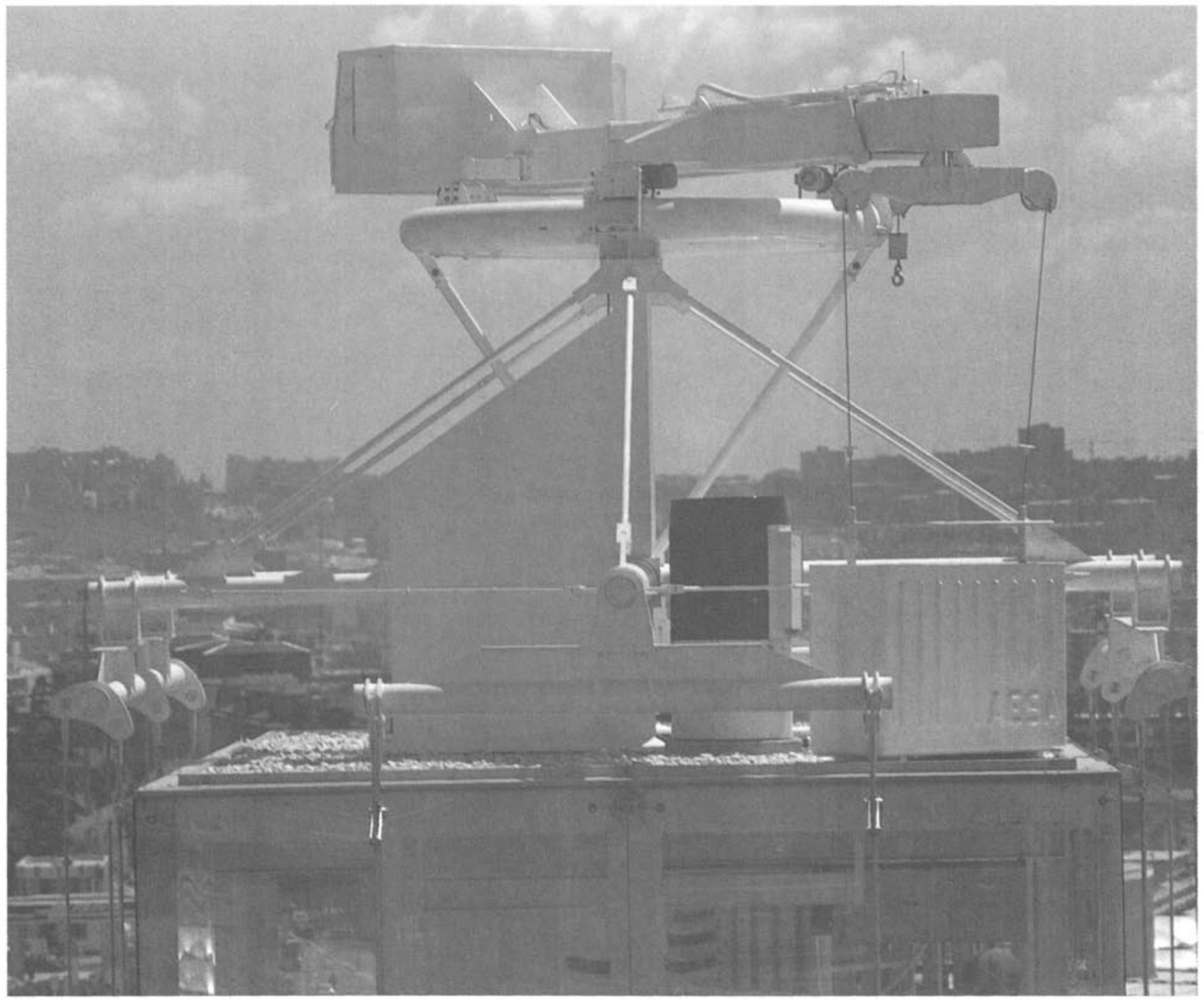

Estructura que soporta en voladizo los pescantes de cuelgue de las fachadas, y también apoyo de grúas polares.
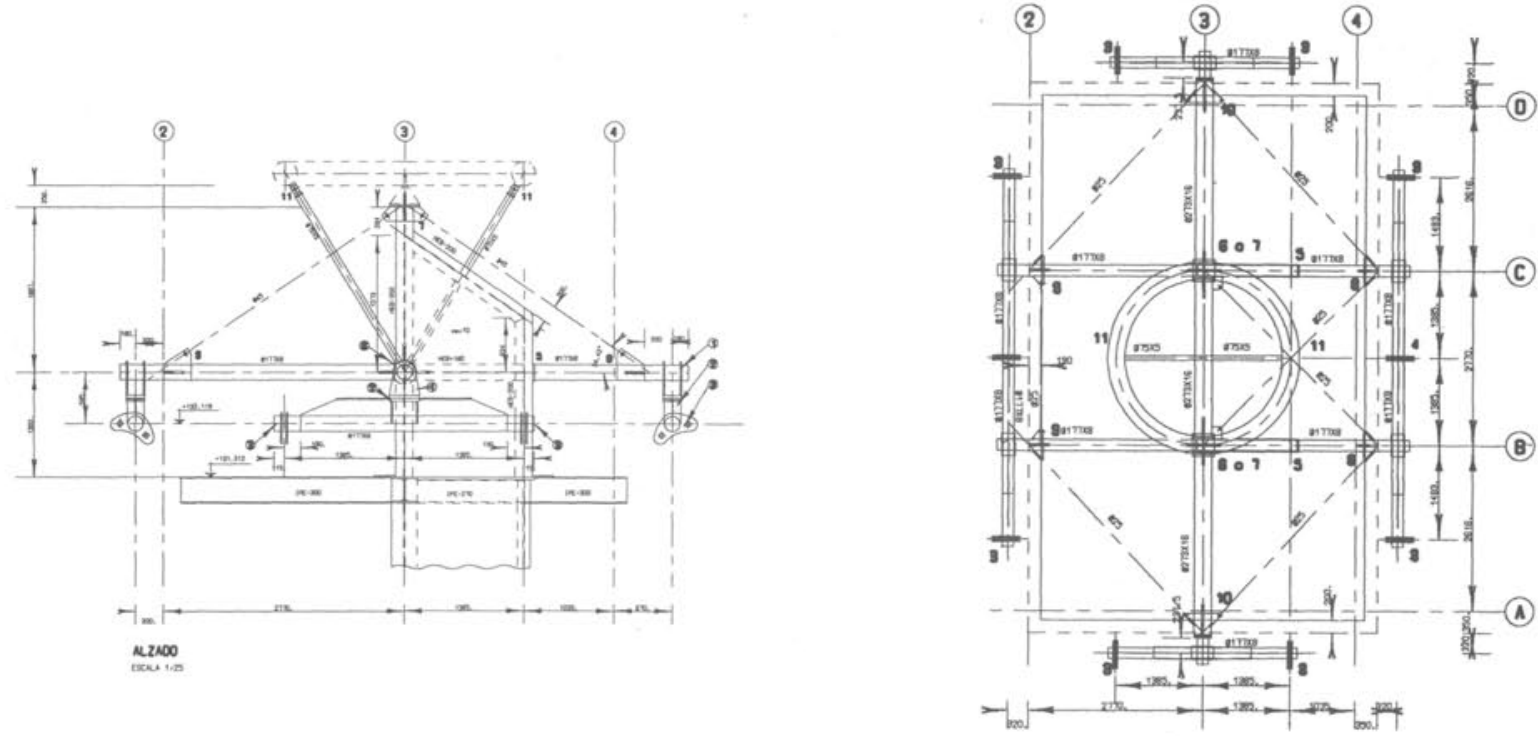

PLANTA ESTRUCTURA SOERE CUBIERTA Esche 1 150 


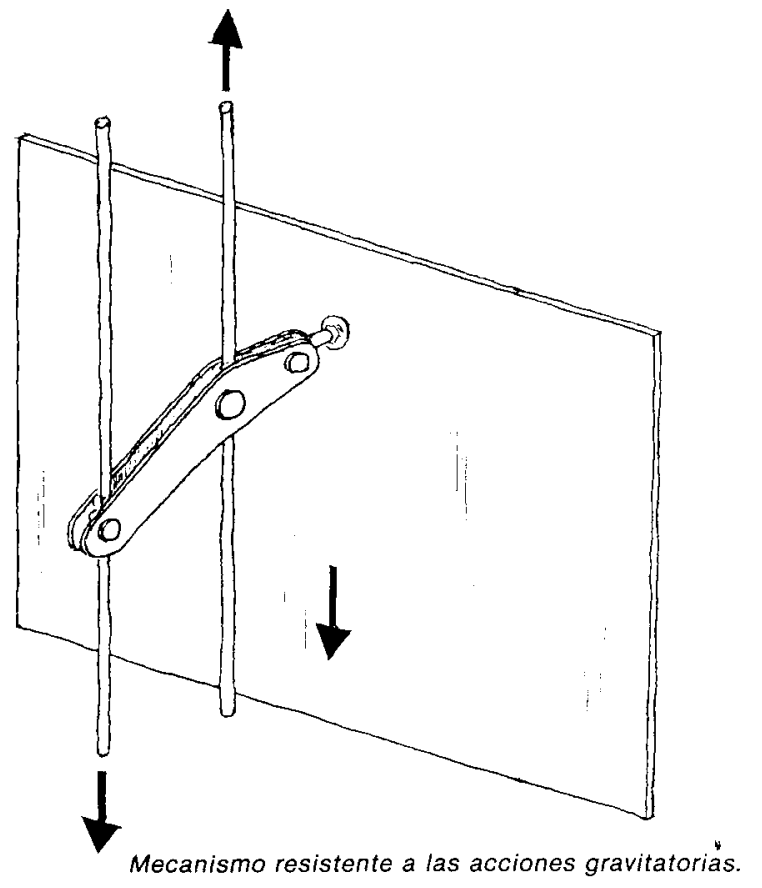

FACHADAS DE VIDRIO

Indudablemente, la mayor singularidad de las torres reside en la solución adoptada para sus fachadas, totalmente de vidrio estructural, transparentes y libres de cualquier tipo de carpintería de soporte.

\section{Materiales}

Los únicos materiales que intervienen en la configuración de la fachada son vidrio y silicona estructurales, y acero inoxidable:

- Vidrio en paneles rectangulares de $12 \mathrm{~mm}$ de espesor, cuyas dimensiones se ajustan a la modulación de las torres y de las diferentes fachadas, con unos valores aproximados de $3.000 \mathrm{~mm}$ por $1.800 \mathrm{~mm}$.

- Acero inoxidable en las barras verticales y en las piezas especiales de suspensión de los paneles de vidrio, así como en los elementos interiores de conexión horizontal con la estructura resistente de las torres.

- Silicona estructural como único material de enlace entre paneles de vidrio, con una función resis. tente al colaborar en la redistribución de esfuerzos entre paneles adyacentes bajo las acciones mecánicas (gravitatorias y eólicas) y térmicas, no homogéneas.

\section{Mecanismos resistentes}

Una de las principales limitaciones del diseño del revestimiento de vidrio reside en la necesidad de evitar

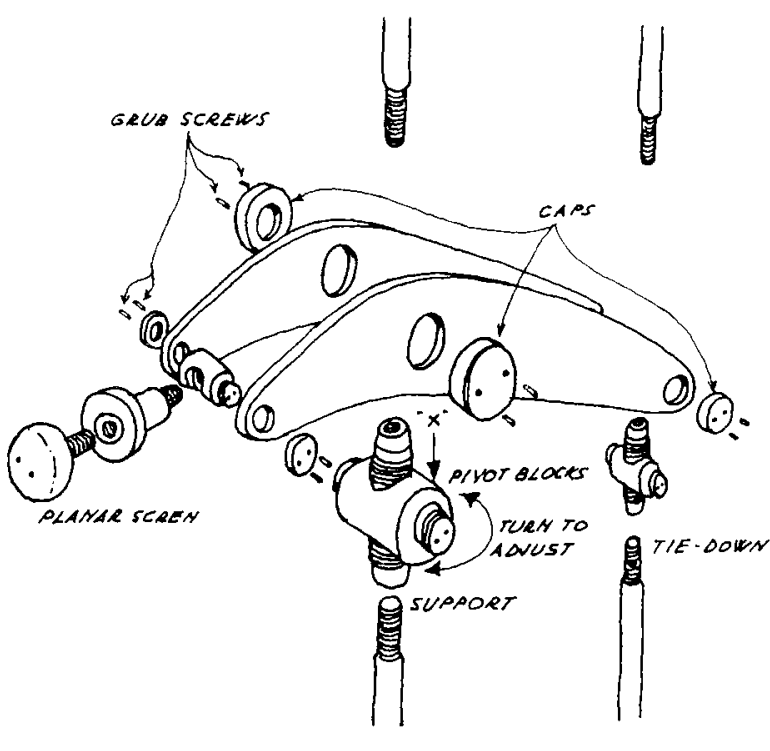

Despiece del soporte de vidrio.

concentraciones de tensiones en los paneles. Para lograr este objetivo la organización mecánico-resistente de la fachada se descompone en dos conjuntos diferenciados:

- mecanismo resistente a las acciones verticales de origen gravitatorio y térmico, y

- mecanismo resistente a las acciones horizontales de origen eólico.

Las cargas verticales debidas al peso propio de cada uno de los paneles de vidrio se suspenden individualmente de la estructura de cubierta mediante una pieza de acero inoxidable (con forma de balancín) unida a través de una rótula al vidrio y conectada a dos barras verticales situadas en el exterior de las torres. Esta duplicidad de las barras de suspensión mantiene el vidrio libre de tensiones de flexión significativas. Cada panel de vidrio va suspendido de un único punto, situado en la parte superior de su eje vertical.

La barra más próxima al plano del vidrio (a $200 \mathrm{~mm}$ de distancia) es el elemento de cuelgue, propiamente dicho, que refiere las cargas verticales a la estructura de cubierta. Su diámetro es de $28 \mathrm{~mm}$ y es de acero inoxidable Duplex (coeficiente de dilatación lineal 12 E-6).

La barra exterior (a $300 \mathrm{~mm}$ de la anterior) actúa como anclaje compensatorio de la excentricidad de las fuerzas y traslada a la base de la torre la reacción ascendente que soporta. Su diámetro es de $20 \mathrm{~mm}$, y es de acero inoxidable de calidad S 316 (coeficiente de dilatación lineal 17 E-6). 


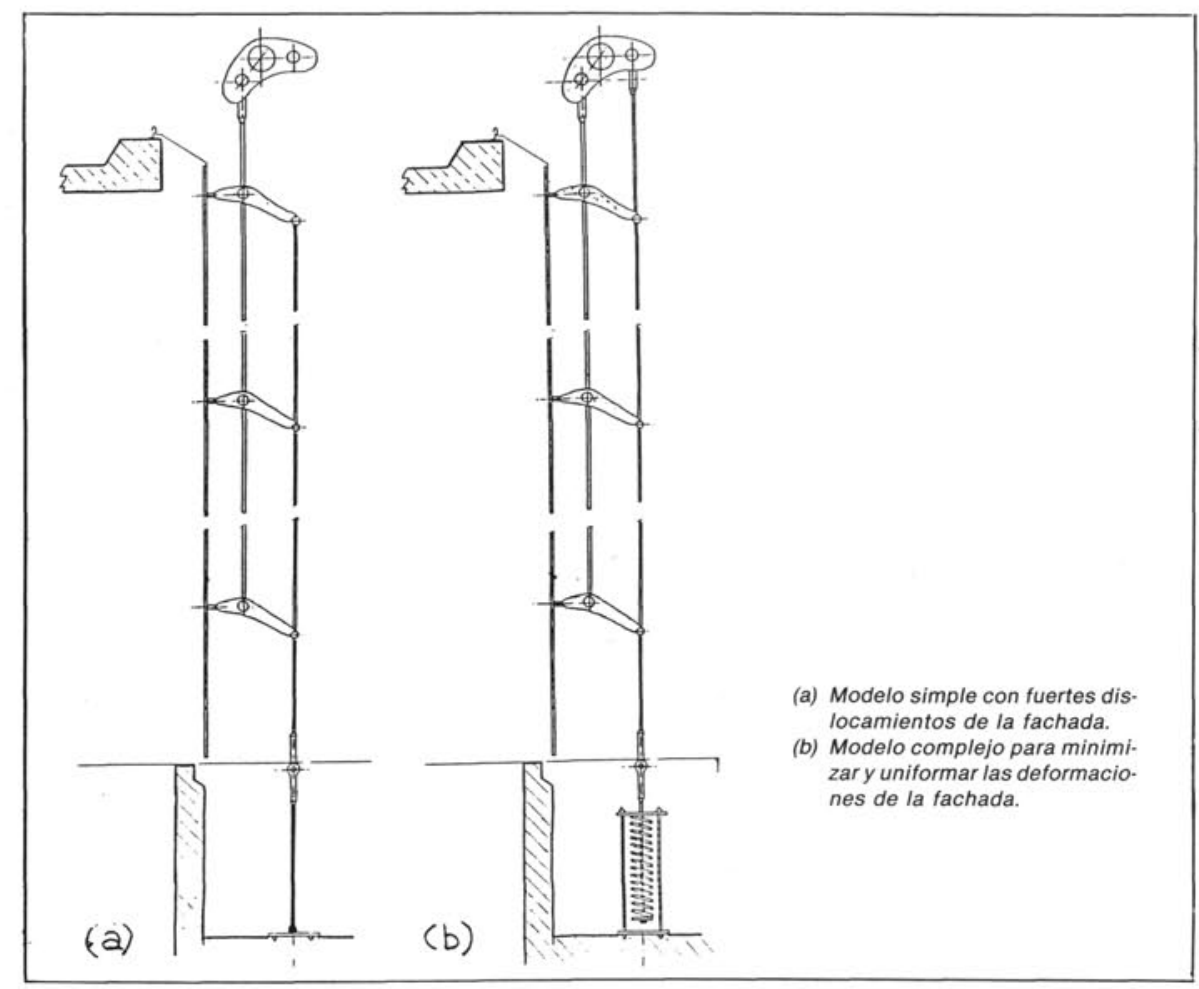

Muelle en la base del edificio conectado al extremo de la barra de preten. sado de fachada.

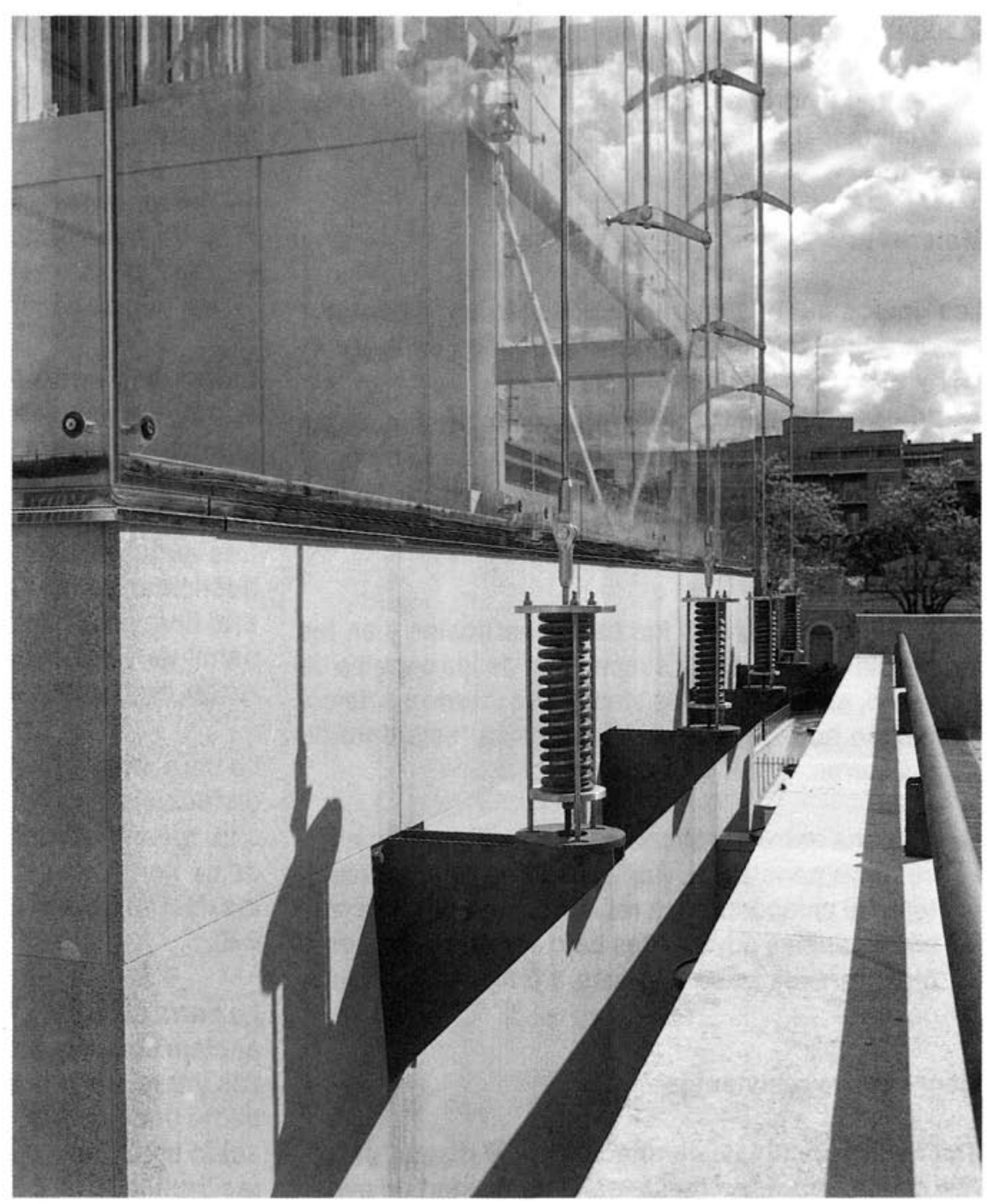




\section{Efectos térmicos}

Bajo los efectos térmicos, la fachada en su conjunto debe deformarse verticalmente con la máxima libertad con respecto a los restantes elementos de la torre.

Las variaciones de temperatura dan lugar a dilataciones y contracciones de la estructura principal, de los paños de vidrio y de las barras de suspensión, siendo diferentes los coeficientes de dilatación lineal de cada uno de estos elementos.

También se producen deformaciones diferenciales entre cada una de las partes como consecuencia de gradientes de temperatura entre la estructura interior $y$ la estructura de cuelgue, $y$ entre unas zonas y otras de la propia fachada, provocadas por el soleamiento parcial de la misma.

Las pequeñas variaciones de geometría entre los paneles de vidrio se disipan en las juntas existentes entre los mismos con redistribuciones tensionales gracias al sellado con silicona estructural. Sin embago, ante diferenciales térmicos más importantes, para poder minimizar y controlar estas deformaciones es preciso dotar al sistema de cuelgue, antes descrito, de algunos mecanismos adicionales de respuesta.

En el supuesto más simple, barra de suspensión unida a la cubierta y barra de compensación anclada exclusivamente a la base, un diferencial térmico entre el interior (estructura principal) y el exterior (barras de suspensión) da lugar a dislocamientos entre los paneles de vidrio de magnitud excesiva. Una mayor temperatura exterior hace alargarse a la barra de suspensión hacia abajo y a la de compensación hacia arriba, ocasionando un significativo descuelgue de los paños de fachada.
Para suprimir el efecto desfavorable anterior y reducir los movimientos de la fachada, además de aprovechar la diferencia entre los coeficientes de dilatación lineal del acero inoxidable de ambas barras de cuelgue, se introducen las siguientes acciones sobre la ba. rra de compensación:

- conectar el extremo inferior a la base del edificio mediante un muelle (de $610 \mathrm{~mm}$ de longitud libre, $25,4 \mathrm{~mm}$ de diámetro y con un módulo de deformación de $65 \mathrm{~mm} / \mathrm{t}$ ),

- enlazar el extremo superior con la estructura de cubierta, y

- aplicar un pretensado inicial con ayuda del propio resorte de la base, de tal forma que se mantenga un permanente estado de tracción, incluso en las condiciones más extremas de sobrecalentamiento de las barras. Se han aplicado $6 \mathrm{kN}$ de pretensado a cada barra para permitir un movimiento térmico del muelle de $40 \mathrm{~mm}$, sin que desaparezca el pretensado.

El pretensado de la barra de compensación es necesario para reducir los movimientos del vidrio, asegurando que tanto la barra de cuelgue como la de compensación se expanden hacia abajo cuando se incrementa su temperatura.

El fenómeno térmico antes considerado, mayor temperatura en el exterior de la torre que en su interior, se traduce en estas circunstancias en un alargamiento de ambas barras hacia abajo, reduciéndose la dimensión del muelle por pérdida parcial del pretensado inicial; la deformación del faldón de vidrio es menor, así como los dislocamientos entre paneles. 

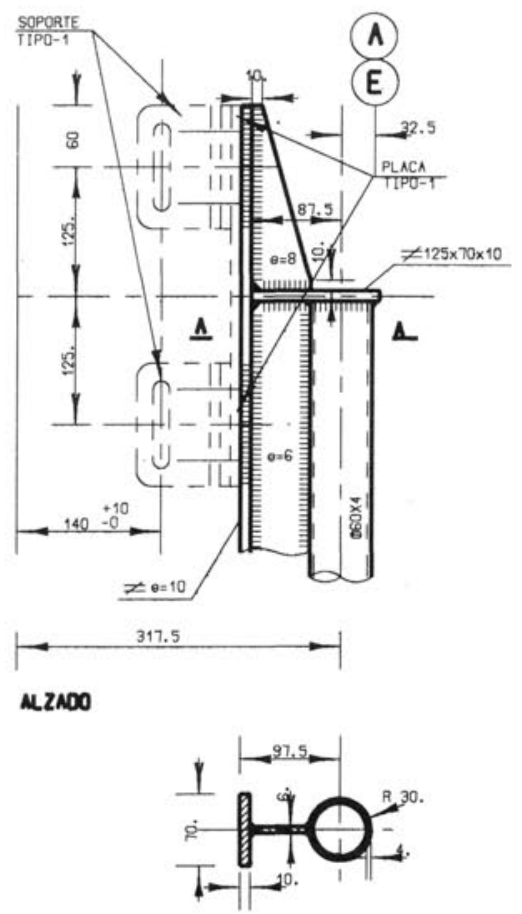

PLANTA seccion A-A

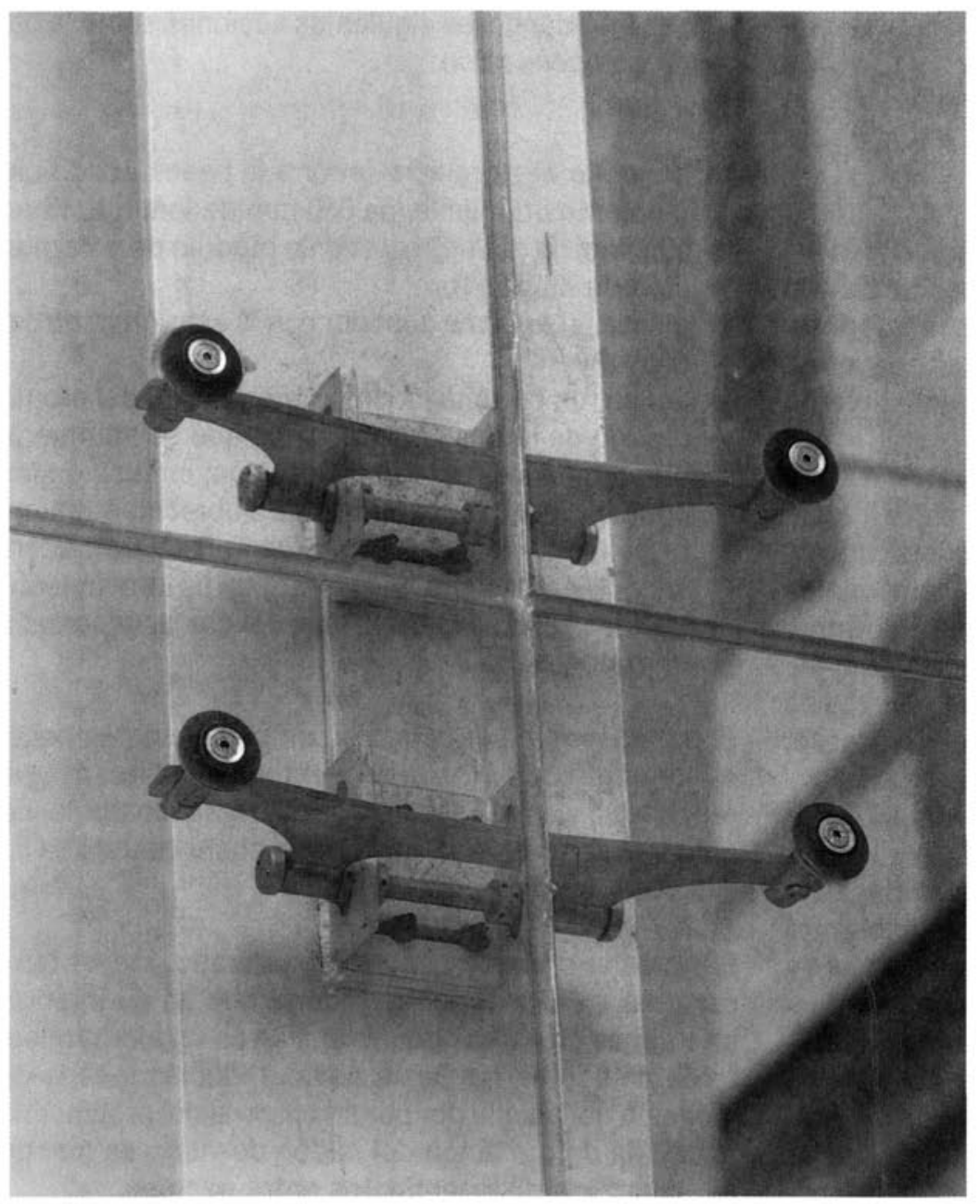

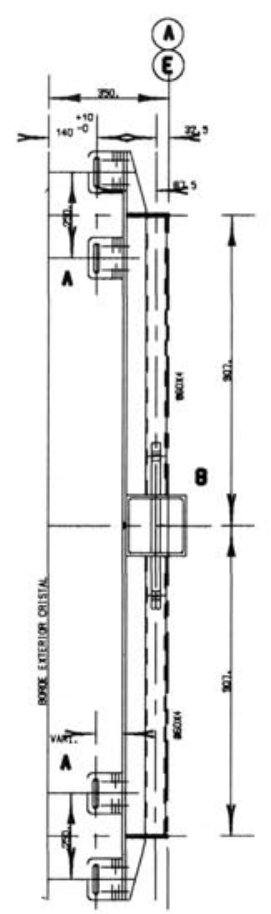

OETMLLe -2- 10 unomoses

$\operatorname{esch} 4+10$

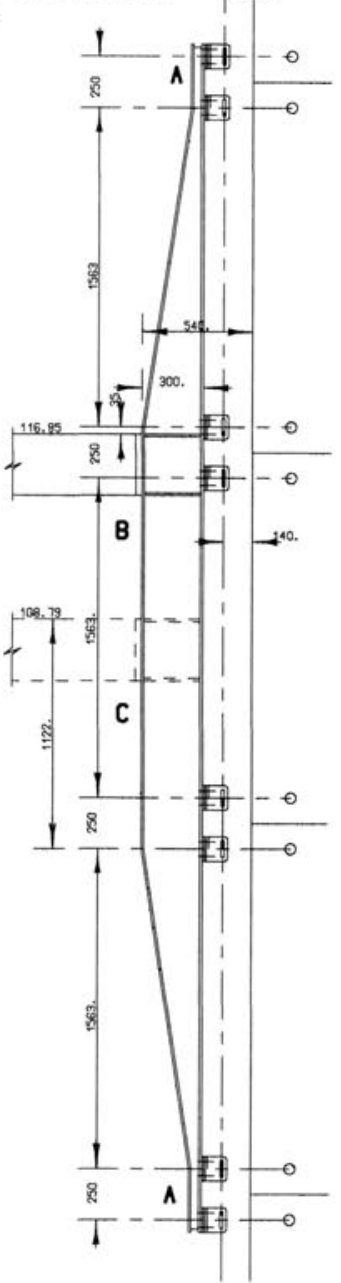

DETALLE-9- NIVELES

108. 79 Y 116.95 (4 Unionoes) ESCALA 1,20 


\section{Efectos del viento}

Las fuerzas horizontales de viento que actúan sobre los paneles de vidrio son transmitidas mediante piezas de fijación, solidarias al vidrio, a la estructura principal de las torres. Estas piezas de acero inoxidable se sitúan en las cuatro esquinas de cada uno de los paneles de vidrio.

El vidrio es particularmente vulnerable a las tensiones locales de flexión producidas por los elementos de fijación, y debe evitarse que las piezas de fijación estén transmitiendo el peso del vidrio directamente a la estructura de la torre (Pág. 56).

Para minimizar la coacción que estas piezas producen al libre movimiento vertical del conjunto de la fachada, su conexión a la estructura se realiza mediante elementos verticalmente ranurados que liberan los movimientos deseados.

\section{Equipo técnico}

$\begin{array}{ll}\text { Arquitectos: } & \begin{array}{l}\text { José Luis Íñiguez de Onzoño } \\ \text { Antonio Vázquez de Castro }\end{array} \\ \text { Aparejador: } & \text { Antonio Palomo Herranz } \\ \text { Colaboradores: } & \text { IAN RITCHIE ARCHITECTS } \\ & \text { OVE ARUP and PARTNERS } \\ & \text { HUARTE, s. a. (Oficina Técnica) } \\ \text { Constructor: } & \text { HUARTE, s. a. } \\ \text { Propiedad: } & \text { Ministerio de Cultura }\end{array}$

Tomás Tabera

Fotografias: Foto Estudio Alarcón.

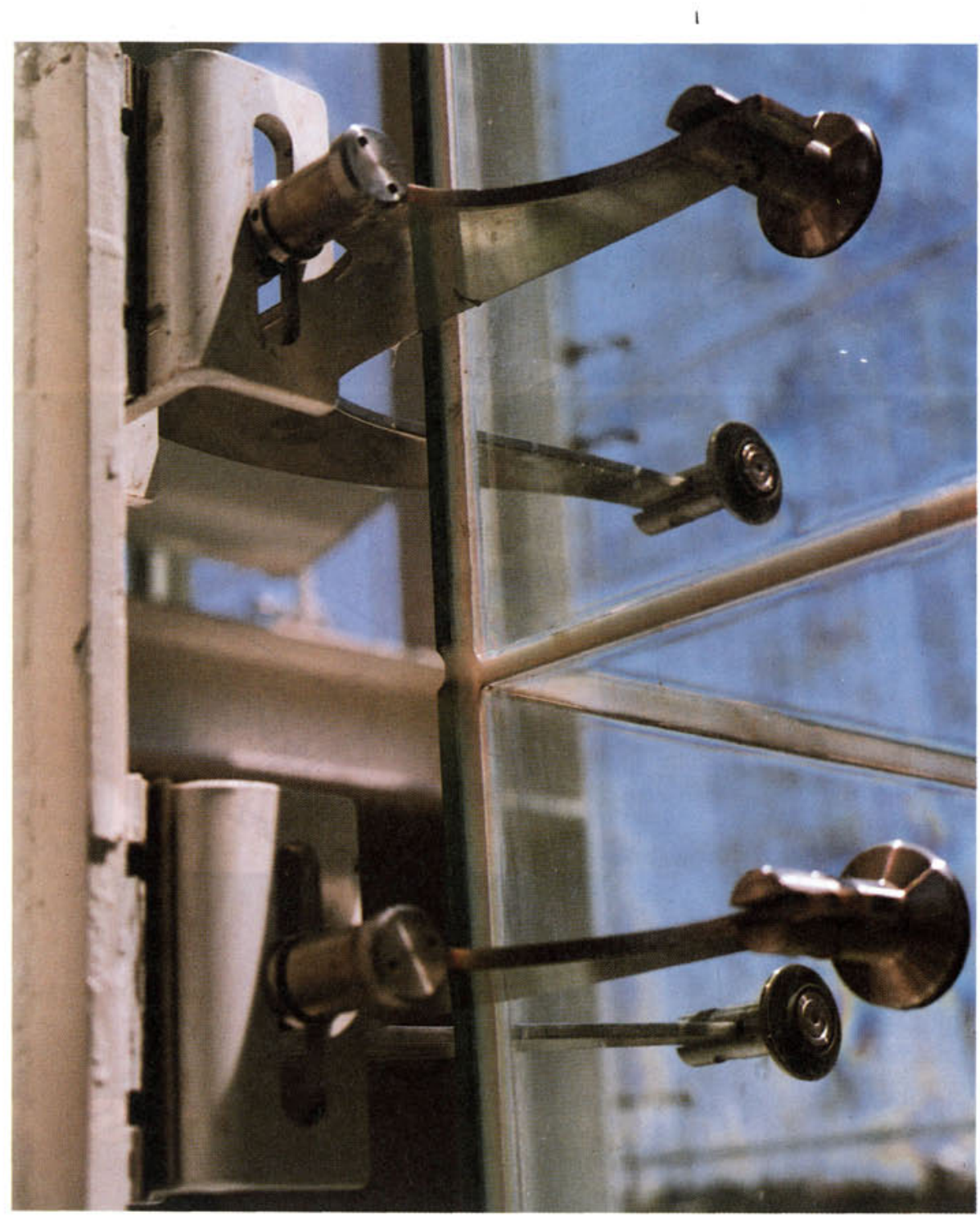

